\title{
CASCADED STRUCTURES FOR BLIND SOURCE RECOVERY
}

\author{
Khurram Waheed and Fathi M. Salam \\ Circuits, Systems and Artificial Neural Networks Laboratory \\ Michigan State University \\ East Lansing, MI 48824-1226
}

\begin{abstract}
Blind Source Recovery (BSR) is an interesting autonomous and unsupervised stochastic adaptation problem that includes the well-known blind adaptive problems of Blind Source Separation (BSS), Deconvolution (BSD) and Equalization (BSE). BSR includes also the nonlinear case and hences focuses on reproducing or estimating the source signals even if environment identifiction is not achieved. A number of outstanding research contributions have been made in this field, however, the issues of application are still in their infancy. Most of the BSR algorithms have characteristics, which make them suitable for a particular subclass of problems. In order to develop a generalized source recovery framework and yet achieve optimal performance in all cases, there is a need to explore further architectural and/or algorithmic domains. In this paper, we approach this goal in the architecture domain by focusing on the use of cascaded structures for BSR. The paper discusses the need, choice, possible forms and properties of several cascaded structures. Some illustrative simulations have been included to highlight the advantages of some of the proposed structures.
\end{abstract}

\section{Introduction}

Interest in the use of cascaded structures for the blind recovery problems is warranted by a number of practical situations. As cascaded structures, we would like to examine the interrelationship of having more than one demixing systems cascaded (in series, see Fig. 2) for the purpose of recovering the sources from environments that may include transients, convolution and possible nonlinearity. Before focusing on the topic of series interconnect of two (or more) demixing structures, please note that other possible inter-connection formats (see Fig. 1) like the parallel and the feedforward-feedback structures lead to added complexities. In the parallel case, because of inherent permutation ambiguity in each system, the combined output of the network might be useless. However, note that this issue can be avoided in the semi-blind cases where some a priory information of the sources may be known. While for the feedforward-feedback case, the two systems become coupled which results in computationally more expensive update laws, if they can be developed at all. Furthermore, the stability issues for such systems are hard to analyze. In this paper, we restrict our choice to having more than one demixing system in a series connection only.

\subsection{Why Cascaded Structures?}

The cascaded structures are useful in a number of practical situations, some of these are discussed below

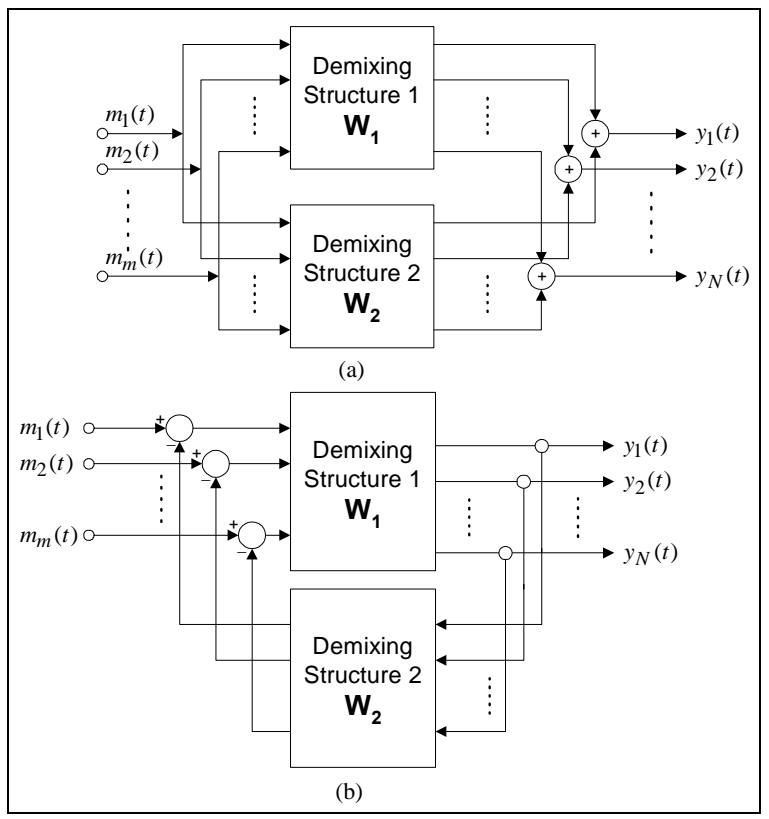

Figure 1. Other possible interconnects of demixing structures (a) Parallel structure (b) Feedforward-Feedback structure

1. The first system may not be a demixing system at all but another data processing system, which collects useful information from the incoming observation mixtures that may aide or enhance the overall recovery of sources, examples include having a beam-former before the demixing system to take care of undesired problems such as permutation of sources by the demixing/recovering network. This is extremely useful for situations where the source exhibits restricted spatial diversity or motion, e.g., a presenter in a conference room etc. Other such pre-processing steps may include conditioning of sources such as pre-whitening by PCA type algorithms, equalization of mixture covariances etc.

2. For unknown dynamic convolutive mixing of sources, the structure of the first demixing matrix may not be able to completely recover the sources at all times due to the time varying nature of the problem. Thus necessitating another demixing system to expand/improve the performance of the first system. However, in such cases a sophisticated control mechanism is required for tandem switching or combined operation of these demixing structures. e.g., consider the case of mobile speakers in a room, the changing position of speakers with respect to the recording microphones represent a continuously time varying mixing transfer function, which requires a continuously adapting demixing network where the 


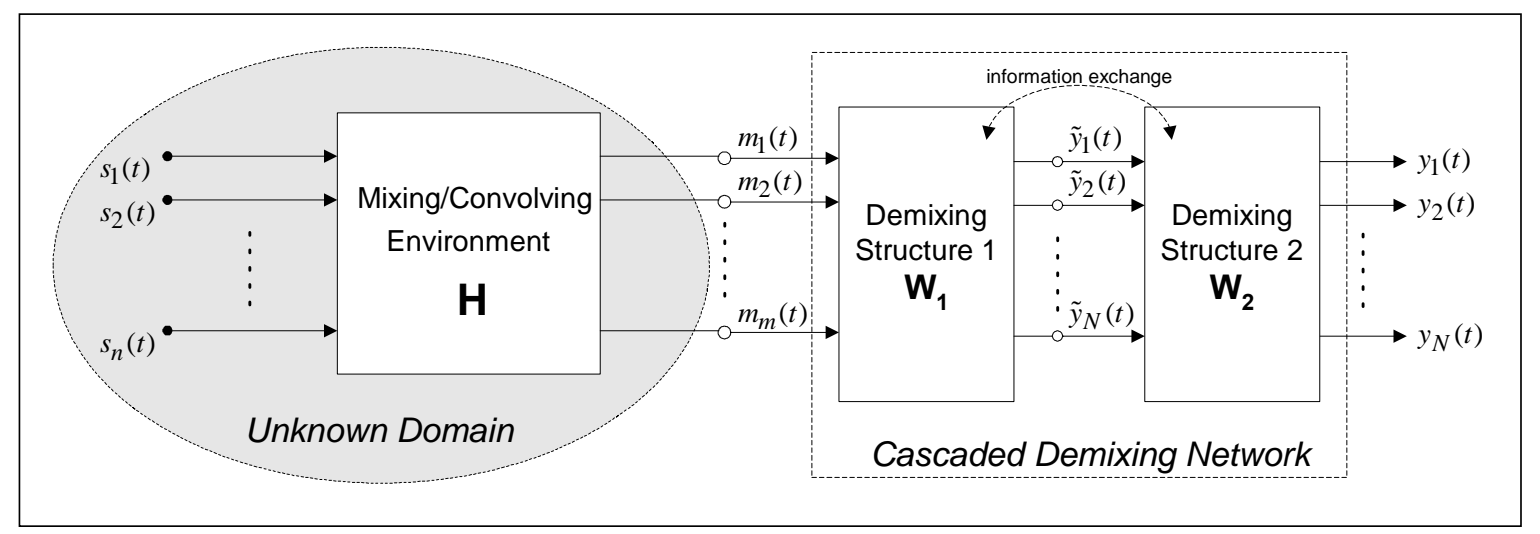

Figure 2. Cascaded demixing framework comprising of two cascaded structures

demixing system order, type (minimum phase/ non-minimum phase), permutation is also time variables. This may render the first structure to be inadequate during certain time intervals and another system may be required to post process the output of the first system for an improved performance.

3. All demixing algorithms use a score function or a nonlinearity in their update laws. The optimal choice of this nonlinear function requires knowledge of the underlying source distributions. In a completely blind scenario, a score function selected for the BSR network may not be optimal and may at the end result in an incomplete recovery of sources. In such cases, a second stage will be required to cater for this nonlinearity mismatch. Consider the example of a linear deconvolution problem where a score function based on the higher order statistics [3] is chosen for the demixing structure 1. The setup is suited to the needs of the minimum phase demixing of communication channels, where the dominant source distribution is sub-gaussian. In presence of a spurious source with gaussian or super-gaussian distribution, the network will converge to a solution, where the sources are deconvolved but not completely separated. In this case a second blind source separation stage may be required to take care of this remnant instantaneous mixing (see simulation I).

4. Most real world convolutive mixing (chain of instantaneous mixing filters) problems constitute nonminimum phase mixing environments. While the characterization, performance and computational efficiency of minimum phase BSR algorithms make them more attractive for implementation [4,5,7]. It is well known that a non-minimum phase system can be dichotomically split into a minimum phase system and a corresponding non-minimum phase all-pass filtering structure. This suggests the possibility to model a nonminimum phase environment as a cascade of a non-minimum phase and a minimum phase demixing system. Note that the order of the all-pass non-minimum phase filter is normally much smaller than the original non-minimum phase system. This suggested cascaded structure can effectively minimize ISI as compared to the use of non-minimum phase demixing network alone.

5. Due to the variety in the application and convergence characteristics of several different BSR algorithms, different algorithms can be chosen for specific recovery tasks. A complex application may entail specific source recovery requirements, where fusion of more than one algorithm may result in the achievement of desired performance level. Consider, e.g., state space feedforward algorithm $[4,6]$ where the natural gradient upgrade of $C$ and $D$ matrices effectively results in modification of the transmission zeros of the demixing network, while a similar update for the feedback algorithm [6] may result in constrained modification of poles as well as zeros. So a cascaded fusion of the two algorithms may result in a system where the former will try to repeal the effect of environment poles, while the latter will try to latch onto the transmission zeros of the network. This effect may be exploited to obtain a compact demixing network representation.

6. In case of blind extraction of multiple sources by a deflation type procedure, a certain demixing structure is applied iteratively to the observed mixtures to extract and remove sources sequentially [1]. However, note that in this case, the adaptation for a stage starts only after it has terminated for a previous stage and the corresponding source removed from the mixture. This procedure results in a stepwise simplification of the problem.

\section{Problem Definition and Architecture}

Using a two-system cascaded demixing structure as shown in Fig. 2, the overall source recovery is achieved by the combination of the two constituent demixing structures. Let the complete demixing structure is given by W. Assuming both cascaded structures to possess linear dynamics

$\mathbf{W}=\mathbf{W}_{\mathbf{2}} \mathbf{W}_{1}$

The BSR problem requires that $[3,4]$

$\mathbf{W H}=P \Delta$

or

$\mathbf{W}_{\mathbf{2}} \mathbf{W}_{\mathbf{1}} \mathrm{H}=P \Delta$

In the case that $\mathbf{W}_{\mathbf{1}} \mathbf{H}$ is invertible and $\mathbf{W}_{\mathbf{2}}$ is adapted after $\mathbf{W}_{\mathbf{1}}$, the cascaded system $\mathbf{W}_{\mathbf{2}}$ has to satisfy

$\mathbf{W}_{\mathbf{2}}=P \boldsymbol{\Delta}\left(\mathbf{W}_{\mathbf{1}} \mathbf{H}\right)^{\dagger}$

where 
$\left(\mathbf{W}_{\mathbf{1}} \mathbf{H}\right)^{\dagger}$ represents the generalized inverse of $\mathbf{W}_{\mathbf{1}} \mathbf{H}$ in the systems sense

Comparing (2.3) with the requirements on a linear demixing network [3,4], we observe that $\mathbf{W}_{\mathbf{2}}$ is facing a problem that is identical to having a single demixing structure, but $\mathbf{W}_{\mathbf{1}}$ is a demixing system on its own, which has already partially equalized $\mathbf{H}$. In this case, the demixing system order required for $\mathbf{W}_{\mathbf{1}} \mathbf{H}$ will be much smaller than the original mixing environment $\mathbf{H}$.

In the other case where $\mathbf{W}_{\mathbf{1}}$ takes the form of an information extraction stage, rather than a demixing stage, the original mixtures will be unaltered for $\mathbf{W}_{\mathbf{2}}$, which implies that $\mathbf{W}_{\mathbf{1}}=I$ or some scaling diagonal strcture $\Lambda$, for the mixture observations and in this case (2.3) reduces to the single stage BSR problem $[3,4]$. Moreover, the BSR task is simplified due to the more information available for adaptation of $\mathbf{W}_{\mathbf{2}}$. Whereas if $\mathbf{W}_{\mathbf{1}}$ becomes a pre-processor of data, e.g., a PCA pre-whitening stage for BSS, it simplifies the blind recovery task for $\mathbf{W}_{\mathbf{2}}$, as the input to $\mathbf{W}_{\mathbf{2}}$ is already projected onto orthonormal bases. A preprocessing PCA stage has been widely analyzed for adaptive systems [see 2 and the references therein].

\subsection{Theorem}

For a cascaded structure of two BSR networks with independent state variables (see figure 8.2), the update law for each network is independent of the internal states of the other network.

\subsubsection{Proof}

Consider the two stage cascaded structure as shown in Fig. 2. The input-output relation for the first network is given by

$\tilde{Y}=\mathbf{W}_{\mathbf{1}} M$

where

$\tilde{Y}$ - represents the $n$-dimensional output of the first network.

$M$ - represents the $m$-dimensional input to the network (observed mixtures)

For the second network, the input-output relationship is given by

$Y=\mathbf{W}_{\mathbf{2}} \tilde{Y}$

where $Y$ - represents the $n$-dimensional output of network 2 .

Combining (2.4) and (2.5) in an augmented system, we have

$\left[\begin{array}{c}\tilde{Y} \\ Y\end{array}\right]=\left[\begin{array}{cc}\mathbf{W}_{1} & 0 \\ 0 & \mathbf{W}_{\mathbf{2}}\end{array}\right]\left[\begin{array}{c}M \\ \tilde{Y}\end{array}\right]$

Defining $\bar{Y}=\left[\begin{array}{c}\tilde{Y} \\ Y\end{array}\right], \bar{W}=\left[\begin{array}{cc}\mathbf{W}_{\mathbf{1}} & 0 \\ 0 & \mathbf{W}_{\mathbf{2}}\end{array}\right]$, and $\bar{X}=\left[\begin{array}{c}M \\ \tilde{Y}\end{array}\right]$, we can compactly write (2.6) as

$\bar{Y}=\bar{W} \bar{X}$

Using the natural gradient, the update rule for $\bar{W}$ is given by [4]
$\Delta \bar{W}=\eta\left[I-\varphi(\bar{Y}) \bar{Y}^{T}\right] \bar{W}$

Inserting the values of the dummy variables $\bar{W}$ and $\bar{Y}$, we have

$\Delta\left[\begin{array}{cc}\mathbf{W}_{\mathbf{1}} & 0 \\ 0 & \mathbf{W}_{\mathbf{2}}\end{array}\right]=\eta\left[I-\varphi\left(\left[\begin{array}{c}\tilde{Y} \\ Y\end{array}\right]\right)\left[\begin{array}{c}\tilde{Y} \\ Y\end{array}\right]^{T}\right]\left[\begin{array}{cc}\mathbf{W}_{\mathbf{1}} & 0 \\ 0 & \mathbf{W}_{\mathbf{2}}\end{array}\right]$

The update laws for individual systems are given by

$\Delta \mathbf{W}_{\mathbf{1}}=\eta\left[I-\varphi(\tilde{Y}) \tilde{Y}^{T}\right] \mathbf{W}_{\mathbf{1}}$

and

$\Delta \mathbf{W}_{\mathbf{2}}=\eta\left[I-\varphi(Y) Y^{T}\right] \mathbf{W}_{\mathbf{2}}$

while the other terms of (2.9) imply mutual independence of $\tilde{Y}$ and $Y$, which is an expected result (see equation (2.3)) i.e.,

$\varphi(\tilde{Y}) Y^{T}=\varphi(Y) \tilde{Y}^{T}=0$

Therefore, it is observed that using a cascaded structure, the second system has an update law, which only depends on the output of the first system. It is not related to the parameters of the first network.

This results in two possible options to update the parameters of the two systems: sequential update and simultaneous update.

In sequential update, the parameters of the second network are adapted after the parameters of the first network have already been determined. In this case, the second system will be adapted to improve the performance that can be achieved with the first system alone.

In simultaneous update, the parameters for both the networks are simultaneously adapted. This will certainly require a smaller number of iterations as compared to the sequential update. However, this case will occur in situations where we decide to employ a cascaded structure for adaptation right from the onset.

\section{Simulation Results}

In order to highlight some of the points made in section 1.1, some simulated results for BSR are presented. Due to the space limitations, we restrict ourselves to the discussion of results only.

3.1 Simulation I: Deconvolution Network followed by a Separation Network

This simulation presents the results for a $3 \times 3$ IIR mixing environment. The original source signals have multiple source distributions that include super-gaussian, gaussian and subgaussian respectively. The score function for the demixing BSR network is derived based on the higher order statistical GramCharlier expansion [3 and the references therein]. In this case, we observe that the BSR network is able to successfully deconvolve the temporal self and interferer signal echoes. However, the global transfer function (GTF) still is not completely diagonalized but rather represents an instantaneous mixing case. In this case a successive ICA stage, which can be either 
sequentially or simultaneously updated, can take care of the leftover instantaneous mixing. It is evident in Fig. 3 that after the second stage of ICA, the overall GTF is diagonalized, with offdiagonal elements having a negligible variance around zero.

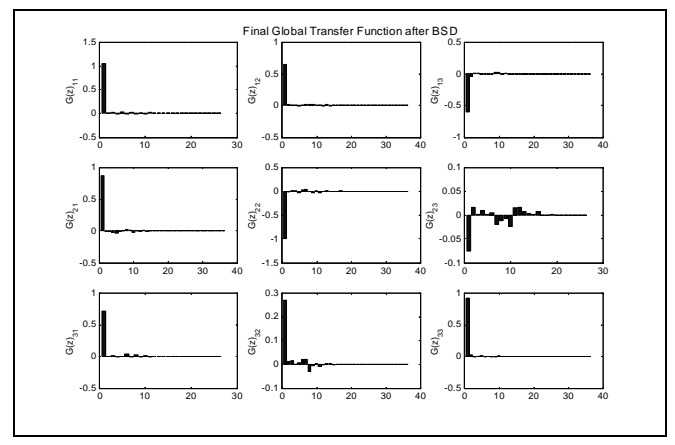

(a)

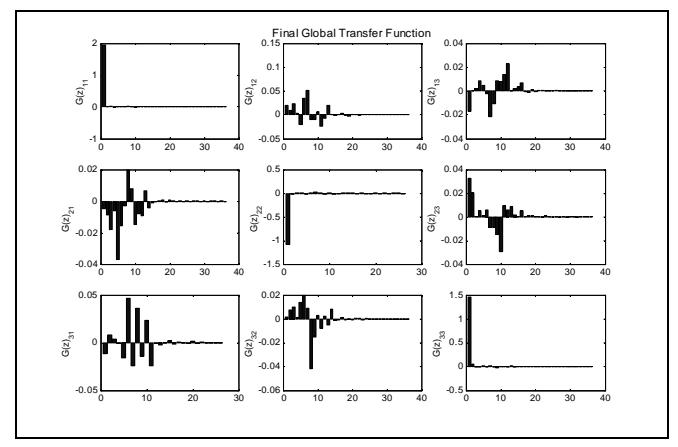

(b)

Figure 3. Global Transfer Function (a) after the BSD stage using a HOS score Function (b) after the cascaded ICA structure

3.2 Simulation II: BSR using a Non-Minimum Phase Network followed by a Minimum Phase Network

For this simulation, the mixing environment is a $3 \times 3$ nonminimum phase IIR filter. Fig. 4 presents the final global transfer function (GTF) matrix using the Non-minimum phase BSR network [7] only and when the demixing is done by a simultaneously updated cascaded network comprising of a nonminimum phase network followed by a minimum phase network. The sources have the same composition as in Simulation I. We observe that the output of the cascaded structure is more delayed (approx. 3 taps), but the following minimum phase network (with 5 taps per element only) improved the separation quality especially for the gaussian distributed source (see $G_{22}(z)$ ). The presented results are after an equal number of instantaneous updates of both networks. It is observed that, if allowed to update, the cascaded structure eventually achieves ISI characteristics better than the non-minimum phase structure alone for all individual components of GTF.

\section{Conclusions}

The issue of using cascaded structures for BSR is formally presented. A detailed discussion on the requirement for such structures has been done. Some promising areas for the pursuit of cascaded BSR structures have been pointed out. Results for a couple of motivating simulation examples are discussed.
However, there is a lot more in this area to be discussed and presented. More results on the topic will be part of subsequent publications by the authors.

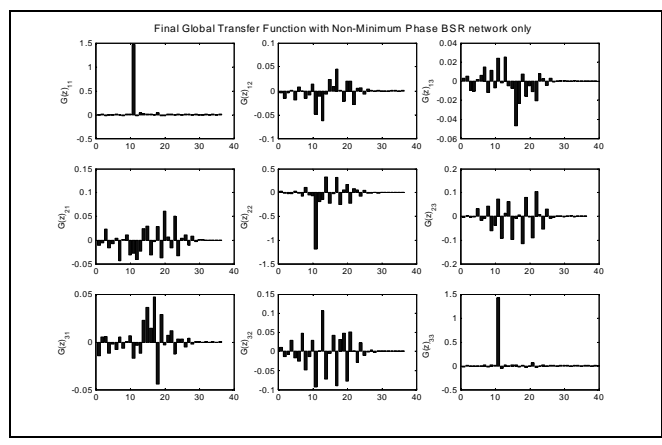

(a)

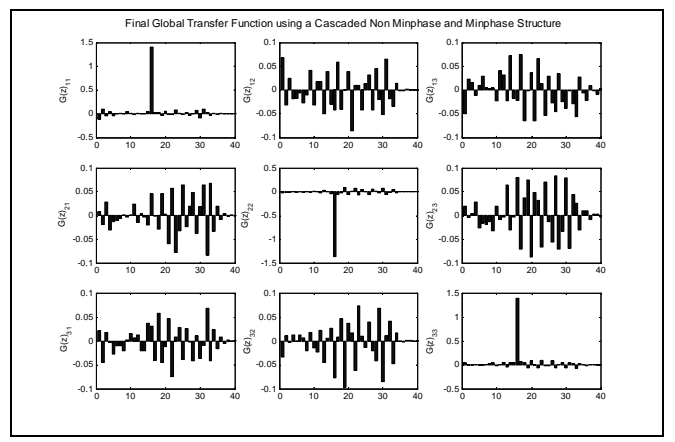

(b)

Figure 4. Global Transfer Function (a) after the Non-minimum phase BSR (b) after the cascaded Non-minimum and Minimum Phase BSR

\section{References}

[1] Choi S. and Cichocki A.: "On-line Sequential Multichannel Blind Deconvolution: A deflation approach" in Proc. IEEE DSP workshop, Utah, August 9-12, 1998. Page(s) 159-162.

[2] LeBlanc J. P. and Fijalkow I.: "Blind Adapted, Pre-Whitened Constant Modulus Algorithm", in IEEE Int'l Conference on Communications, Helsinki, Finland, June 2001.

[3] Salam, F. M.; Gharbi, A. B.; Erten, G.: "Formulation and Algorithms for Blind Signal Recovery", Proceedings of the 40th Midwest Symposium on Circuits and Systems, 1997. Volume: 2, 1998 Page(s): $1233-1236$

[4] Salam F. M., Erten G. and Waheed K.: "Blind Source Recovery: Algorithms for Static and Dynamic Environments"; Int'l Joint Conf. on Neural Networks, July 14-19, 2001Washington, D.C.; Vol. 2, Page(s) 902-907

[5] Waheed K. and Salam F. M.: "Blind Source Recovery: Some Implementation and Performance Issues"; $44^{\text {th }}$ IEEE Midwest Symp. On Circuits and Systems; August 14-17 2001- Dayton Ohio. Vol. 2, Page(s) 580-583

[6] Salam F. M. and Waheed K.: "State Space Feedforward and Feedback Structures for Blind Source Recovery", in $3^{\text {rd }}$ Int'l Conf. on ICA and BSS, December 9-12, 2001 - San Diego, CA; Page(s) 248-253

[7] Waheed K. and Salam F. M.: "State Space Blind Source Recovery For Mixtures Of Multiple Source Distributions"; in Proc. of Intl Symposium on Circuits and Systems ISCAS-2002, May 26-29, 2002 - Phoenix, Az; Vol I, Page(s) 197-200 